University of Wollongong

Research Online

Faculty of Engineering and Information

Faculty of Engineering and Information

Sciences - Papers: Part B

Sciences

2018

\title{
Lattice Boltzmann simulation of flow and heat transfer evolution inside encapsulated phase change materials due to natural convection melting
}

Qi Lin

Dalian University of Technology

Shugang Wang

Dalian University of Technology, shugang@uow.edu.au

Zhenjun Ma

University of Wollongong, zhenjun@uow.edu.au

Jihong Wang

Dalian University of Technology

Tengfei Zhang

Dalian University of Technology

Follow this and additional works at: https://ro.uow.edu.au/eispapers1

Part of the Engineering Commons, and the Science and Technology Studies Commons

Research Online is the open access institutional repository for the University of Wollongong. For further information contact the UOW Library: research-pubs@uow.edu.au 


\title{
Lattice Boltzmann simulation of flow and heat transfer evolution inside encapsulated phase change materials due to natural convection melting
}

\author{
Abstract \\ A comprehensive study of the melting process inside a capsule can potentially take full advantages of \\ latent heat of phase change materials (PCMs). The present study was devoted to the problem of complex \\ interaction of natural convection and melting of PCMs inside a spherical capsule under differen $t$ sizes. \\ The numerical results, simulated by lattice Boltzmann method (LBM), were compared with experimental \\ data and published simulations. The results showed that LBM presented desirable accuracy compared to \\ traditional computational fluid dynamics (CFD) methods. Then, the effects of non-uniform PCM \\ properties, expressed by the solid/liquid thermal diffusivity ratio, on the melting rate were found to be \\ nonlinear in different melting stages. The non-dimensional fully melting time reduced with the increase of \\ the surface temperature and the capsule size, and the former compared to the latter could have a greater \\ influence on the melting rate. Moreover, the non-dimensional fully melting time reduced when increasing \\ of the capsule diameter at the macro-scale; while there was a near-invariable non-dimensional fully \\ melting time when the capsule size was changed at the micro-scale. The good understanding of the \\ phase change process inside the capsule would provide essential information to develop a multi-scale \\ model of microencapsulated PCM slurries. \\ Disciplines \\ Engineering | Science and Technology Studies

\section{Publication Details} \\ Lin, Q., Wang, S., Ma, Z., Wang, J. \& Zhang, T. (2018). Lattice Boltzmann simulation of flow and heat \\ transfer evolution inside encapsulated phase change materials due to natural convection melting. \\ Chemical Engineering Science, 189 154-164.
}




\title{
Lattice Boltzmann simulation of flow and heat transfer evolution inside encapsulated phase change materials due to natural convection melting
}

\author{
Qi Lin ${ }^{1}$, Shugang Wang ${ }^{1, *}$, Zhenjun $\mathrm{Ma}^{2}$, Jihong Wang ${ }^{1}$, Tengfei Zhang ${ }^{1}$
}

(1. Faculty of Infrastructure Engineering, Dalian University of Technology, Dalian 116024, China; 2. Sustainable Buildings Research Centre, University of Wollongong, Wollongong 2500, Australia)

Abstract: A comprehensive study of the melting process inside a capsule can potentially take full advantages of latent heat of phase change materials (PCMs). The present study was devoted to the problem of complex interaction of natural convection and melting of PCMs inside a spherical capsule under different sizes. The numerical results, simulated by lattice Boltzmann method (LBM), were compared with experimental data and published simulations. The results showed that LBM presented desirable accuracy compared to traditional computational fluid dynamics (CFD) methods. Then, the effects of non-uniform PCM properties, expressed by the solid/liquid thermal diffusivity ratio, on the melting rate were found to be nonlinear in different melting stages. The non-dimensional fully melting time reduced with the increase of the surface temperature and the capsule size, and the former compared to the latter could have a greater influence on the melting rate. Moreover, the non-dimensional fully melting time reduced when increasing the capsule diameter at the macro-scale; while there was a near-invariable non-dimensional fully melting time when the capsule size was changed at the micro-scale. The good understanding of the phase change process inside the capsule would provide essential information to develop a multi-scale model of microencapsulated PCM slurries.

Keywords: Melting; Phase change materials; Natural convection; Spherical capsule; Multi-scales; Lattice Boltzmann method

\footnotetext{
* Corresponding author. Tel.: +086-411-84706407; fax: +086-411-84706407.

E-mail address: sgwangln@aliyun.com Abbreviations:

Phase change material - PCM; $\quad$ Computational fluid dynamics - CFD;

Lattice Boltzmann - LB; $\quad$ Multiple-relaxation-time - MRT

Lattice Boltzmann method - LBM; 


\section{Nomenclature}

\begin{tabular}{|c|c|c|c|}
\hline$C$ & mushy zone constant & S & relaxation matrix for flow field \\
\hline$C_{\mathrm{p}}$ & specific heat of PCM $\left[\mathrm{J} \cdot \mathrm{kg}^{-1} \mathrm{~K}^{-1}\right]$ & $T$ & temperature $\left[{ }^{\circ} \mathrm{C}\right]$ \\
\hline$C$ & lattice speed & $\Delta t$ & unit lattice time \\
\hline $\mathbf{F}$ & total body force $\left[\mathrm{N} \cdot \mathrm{m}^{-3}\right]$ & $\mathbf{u}$ & velocity $\left[\mathrm{m} \cdot \mathrm{s}^{-1}\right]$ \\
\hline$f_{1}$ & volume fraction of liquid & $\mathbf{u}_{\mathrm{s}}$ & velocity for solid phase $\left[\mathrm{m} \cdot \mathrm{s}^{-1}\right]$ \\
\hline$f_{i}$ & density distribution function & Gre & k symbols \\
\hline G & acceleration due to gravity $\left[\mathrm{m} \cdot \mathrm{s}^{-2}\right]$ & $\alpha$ & thermal diffusivity $\left[\mathrm{m}^{2} \cdot \mathrm{s}^{-1}\right]$ \\
\hline$g_{i}$ & enthalpy distribution function & $\beta$ & thermal expansion coefficient $\left[\mathrm{K}^{-1}\right]$ \\
\hline$g^{\mathrm{eq}}$ & equilibrium state of $g_{i}$ & $\rho$ & density $\left[\mathrm{kg} \cdot \mathrm{m}^{-3}\right]$ \\
\hline$H$ & total enthalpy $\left[\mathrm{kJ} \cdot \mathrm{kg}^{-1}\right]$ & $\lambda$ & thermal conductivity $\left[\mathrm{W} \cdot \mathrm{m}^{-1} \mathrm{~K}^{-1}\right]$ \\
\hline$L$ & latent heat of fusion $\left[\mathrm{kJ} \cdot \mathrm{kg}^{-1}\right]$ & $v$ & kinematic viscosity $\left[\mathrm{m}^{2} \cdot \mathrm{s}^{-1}\right]$ \\
\hline $\mathbf{M}$ & transformation matrix & Sul & cripts \\
\hline $\mathbf{M}$ & moment vector in flow field & $i$ & specified lattice direction \\
\hline $\mathbf{N}$ & moment vector in temperature field & $l$ & liquid phase of $\mathrm{PCM}$ \\
\hline $\mathbf{R}$ & relaxation matrix for temperature field & $\mathrm{s}$ & solid phase of PCM \\
\hline
\end{tabular}

\section{1. Introduction}

amount of energy within a tiny temperature variation, and thus has been applied to various fields, such as building products [1-4], electronics cooling [5-7], and solar energy storage [8-10]. In recent years, the encapsulated PCM (EPCM) and its slurry serve as a way to enhance 
discharging/charging rate of the PCM by increasing the specific surface area. So far, encapsulated PCM and its slurry have been successfully applied in waste heat recovery [11], cooling systems [12, 13], and ground source heat pump systems [14].

In terms of the scale, the encapsulated PCM can be divided into macro-capsule, micro-capsule and nano-capsule with diameters of $1 \mathrm{~mm}$ to greater than $1 \mathrm{~cm}$, from $1 \mu \mathrm{m}$ to $1 \mathrm{~mm}$, and less than $1 \mu \mathrm{m}$, respectively $[15,16]$. In terms of the geometry, the encapsulated PCM can be classified into sphere, cylinder, and irregular shapes. In terms of the morphology, the encapsulated PCM can be categorized into mononuclear, polinuclear, matrix, and multi-wall [17]. In addition, there are two kinds of the melting mechanisms called unconstrained melting and constrained melting $[18,19]$. The solid PCM will sink down due to gravity with direct contact melting at the bottom of the sphere during the unconstrained melting, while, in the constrained melting, the solid PCM is prevented from sinking to the bottom of the sphere. Different experimental and numerical studies have been performed to investigate PCM behaviors. The difference between the unconstrained melting process and constrained melting process was investigated via experiment methods under different surface temperatures and initial sub-cooling conditions [19]. Some scholars [18, 20, 21] used the conventional computational fluid dynamics (CFD) method to reproduce the constrained melting process inside the spherical capsule, and further analyze the effects of surface temperature, initial condition, and buoyancy-driven on the melting rate. However, the conventional CFD method would suffer from an empirical mushy zone constant, which was recommended between $10^{4}$ and $10^{7}[21,22]$. In contrast, the lattice Boltzmann (LB) model with the volumetric LB scheme can avoid the empirical mushy zone constant with no free parameters when it is used for simulating the melting process $[23,24]$. The 
evolution of temperature and mushy zone by using the lattice Boltzmann method (LBM) was investigated in various cavities such as square cavity [25-27], semicircle enclosure [28], an annulus between two coaxial vertical cylinders [29], and more complex structures [30, 31].

By mixing the encapsulated PCM and heat transfer medium like water, a functional thermal fluid called microencapsulated PCM slurry can be obtained. This slurry offers attractive opportunities for thermal energy transportation and heat transfer enhancement of heat exchangers [32]. The complex thermophysical properties and phase change behavior of the slurry made it difficult to predict the heat transfer of the slurry precisely. So far, many groups [33-38] presented various numerical models with a large amount of assumptions to analyze the heat and mass transfer with phase change in the microencapsulated PCM slurry. These assumptions included a uniform temperature inside the capsule, a single-phase non-Newtonian fluid instead of two-phase slurry, etc. However, the uniform temperature assumption made the melting rate present a linear decline inside of a non-linear trend when the thermal conductivity of the PCM was low. It was because this assumption ignored the drawdown of the melting rate caused by the inhibition of the low thermal conductivity of the PCM, resulting in a deviation between numerical results and experimental data [39]. To the best of our knowledge, based on the correlative algorithm [40, 41], it is reasonable to construct a multi-scale model without the above assumptions, which can provide a result that is more close to the reality. The main idea of the multi-scale model is an improved traditional slurry model by coupling the phase change information of the encapsulated capsules with heat transfer model of the slurry. Thus, it is critical to predict the behavior of the phase change inside capsules.

In our previous work [42], the constrained melting process inside a spherical capsule was 

numerically investigated, and the simulations were then compared with the experimental data. The results showed that the effect of natural convection on the melting process decreased as the capsule size was decreased, and even could be ignored if the capsule diameter was less than 3 mm. In this study, the flow and heat transfer evolution of the PCM inside a spherical capsule was further simulated by using an enthalpy-based LBM. The numerical results were compared with the published simulation and experimental data. The effects of various parameters, such as surface temperature, capsule size, and non-uniform properties of the PCM, on the phase change process were analyzed. The results and their dimensionless forms were discussed in order to investigate the above influences of various parameters under different scales. These results would be useful to study microencapsulated PCM slurries on the heat transfer with phase change processes..

\section{Methodologies and Models}

\subsection{Geometrical model}

\section{The geometrical model considered here is a spherical capsule filled with a solid PCM at an} initial temperature $T_{\text {ini, }}$ which could be a temperature lower than the PCM melting temperature $T_{\mathrm{m}}$. For time $t>0$, the surface temperature of the capsule $T_{\text {surf }}$ was abruptly raised to a high value and the melting process commenced.

Note that the size of microcapsule is so small that the visualization of the melting patterns and the temporal evolution of the local temperature are hard to be measured through experiments. In this study, a spherical capsule with an internal diameter of $101.66 \mathrm{~mm}$ was presented to compare 

monitor the temperature next to the shell. The distance between points $\mathrm{E}$ and I was $50 \mathrm{~mm}$.

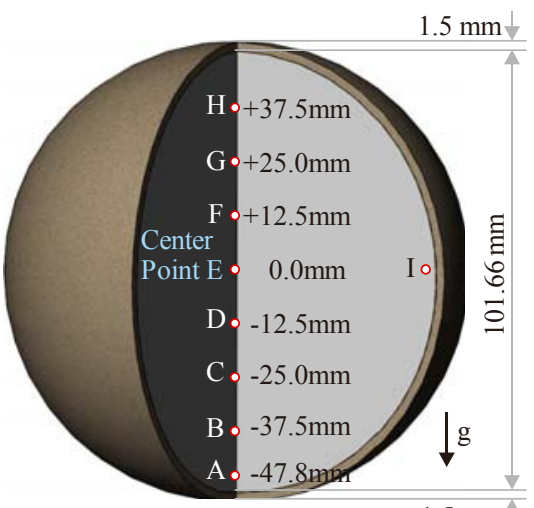

Fig. 1 Spherical capsule and its section with different measurement points [19]. "+" and "-” values mean above

104 For the experimental data used $[19,20]$, the initial temperature was $27.2^{\circ} \mathrm{C}$, which was $1^{\circ} \mathrm{C}$

105 lower than the PCM melting temperature. The surface temperature was $40^{\circ} \mathrm{C}$. Other properties of

Table 1 Thermophysical properties of n-octadecane [19, 20]

\begin{tabular}{ll}
\hline Parameter & Value \\
\hline Melting temperature & $28.2^{\circ} \mathrm{C}$ \\
Density & $772 \mathrm{~kg} \cdot \mathrm{m}^{-3}$ \\
Kinematic viscosity & $5 \times 10^{-6} \mathrm{~m}^{2} \cdot \mathrm{s}^{-1}$ \\
Specific heat & $2330 \mathrm{~J} \cdot \mathrm{kg}^{-1} \mathrm{~K}^{-1}$ \\
Thermal conductivity & $0.1505 \mathrm{~W} \cdot \mathrm{m}^{-1} \mathrm{~K}^{-1}$ \\
Latent heat of fusion & $243.5 \mathrm{~kJ} \cdot \mathrm{kg}^{-1}$ \\
Thermal expansion coefficient & $0.00091 \mathrm{~K}^{-1}$ \\
\hline
\end{tabular}




\subsection{Total enthalpy-based lattice Boltzmann model}

\subsubsection{Assumptions}

In this study, a total enthalpy-based lattice Boltzmann (LB) model [23, 24] was used to simulate the solid-liquid phase change heat transfer in a spherical capsule. It consists of an incompressible LB model for the velocity field and an enthalpy-based LB model for the temperature field. The moving solid-liquid phase interface was implicitly tracked through the liquid fraction, which can be obtained when the energy equation of PCM was solved. To simplify the physical problem, the following assumptions were made for the solid-liquid phase change heat transfer coupled with natural convection inside the spherical capsule.

a) Both the liquid and solid phases are homogeneous and isotropic, and thus their thermophysical properties are taken to be constants;

b) The density differences between solid and liquid phases are ignored;

c) Effective thermophysical properties are used to describe the PCM properties during the melting process, which are depended on the liquid fraction, specific heat and thermal conductivity;

d) Thermophysical properties of the PCM are assumed to be constants over the temperature range considered;

e) The density variation of the liquid PCM that drives natural convection is only considered in the body force terms (i.e. Boussinesq approximation) and;

f) The surrounding temperature around the spherical capsule is maintained constant.

Based on the above assumptions, the phase change process inside the spherical capsule could be considered as a symmetrical distribution around the perpendicular line through the center of 


\subsubsection{Axisymmetric flow LB model}

$\mathbf{m}^{*}(r+\Delta r, t+\Delta t)=\mathbf{m}(r, t)-\mathbf{S}\left[\mathbf{m}(r, t)-\mathbf{m}^{\mathrm{eq}}(r, t)\right]+\Delta t(\mathbf{I}-\mathbf{S} / 2) \mathbf{F}_{m}(r, t)$

where the superscript $*$ means the effect of the solid phase that is not considered, $\mathbf{I}$ is the unit

matrix, $\mathbf{M}$ is the transformation matrix for mapping velocity and moment spaces [44], $\mathbf{S}$ is the

follows [45].

$\mathbf{m}^{\mathrm{eq}}=\rho\left(1,-2+3 \frac{\mathbf{u}^{2}}{c}, 1-3 \frac{\mathbf{u}^{2}}{c}, \frac{u_{r}}{c},-\frac{u_{r}}{c}, \frac{u_{z}}{c},-\frac{u_{z}}{c}, \frac{u_{r}^{2}-u_{z}^{2}}{c}, \frac{u_{r} u_{z}}{c^{2}}\right)^{\mathrm{T}}$

$\mathbf{S}=\operatorname{diag}\left(1.0, \quad 1.0, \quad 1.1, \quad 1.0, \quad 1.2, \quad 1.0, \quad 1.2,\left(0.5+\frac{3 v}{c_{\mathrm{s}}^{2} \Delta t}\right)^{-1},\left(0.5+\frac{3 v}{c_{\mathrm{s}}^{2} \Delta t}\right)^{-1}\right)$

$\mathbf{F}_{m}=\left(0,6 \frac{\mathbf{F} \cdot \mathbf{u}}{c^{2}},-6 \frac{\mathbf{F} \cdot \mathbf{u}}{c^{2}}, \frac{F_{r}}{c},-\frac{F_{r}}{c}, \frac{F_{z}}{c},-\frac{F_{z}}{c}, 2 \frac{F_{r} u_{r}-F_{z} u_{z}}{c^{2}}, \frac{F_{r} u_{z}+F_{z} u_{r}}{c^{2}}\right)^{\mathrm{T}}$

$\mathbf{F}=\left(\frac{-2 \rho v u_{r}}{r^{2}}, \quad \rho|g| \beta\left(T-T_{\text {ref }}\right)\right)$

143 where $F_{r}$ and $F_{z}$ are r- and z-components of the total body force $\mathbf{F}$, respectively, $|g|$ is the gravity

144 acceleration. The thermal expansion coefficient $\beta$ reflects the tendency of the PCM to change in 

and sixth components of the moment vector $\mathbf{m}$ in Eq.(1)(1) should be modified [43].

$$
\begin{aligned}
& \left.m_{3}^{*}\right|_{\text {new }}=m_{3}^{*}-\frac{1}{r}\left[1-\left(1+\frac{6 v}{c_{\mathrm{s}}^{2} \Delta t}\right)^{-1}\right] \Delta t^{2}\left[\left(\frac{1}{12}+\frac{v}{2 c_{\mathrm{s}}^{2} \Delta t}\right) m_{1}^{(1)}+0.5 m_{7}^{(1)}\right] \\
& \left.m_{5}^{*}\right|_{\text {new }}=m_{5}^{*}-\frac{1}{r}\left[1-\left(1+\frac{6 v}{c_{\mathrm{s}}^{2} \Delta t}\right)^{-1}\right] \Delta t^{2} m_{8}^{(1)}
\end{aligned}
$$

where $m_{1}^{(1)}, m_{7}^{(1)}$ and $m_{8}^{(1)}$ can be calculated as follows.

$$
m_{i}^{(1)}=\left(m_{i}-m^{\mathrm{eq}}\right) / \Delta t+0.5 F_{i} \quad i=1,7,8
$$

Based on the volumetric LB scheme [24], the velocity field should be calculated over the entire space that includes fluid and solid phases. Then, the effect of the solid phase can be considered by recalculating the density distribution function in Eq.(1)(1) through a linear interpolation as shown in Eq. (9).

$$
f_{i}=f_{l} f_{i}^{*}+\left(1-f_{l}\right) f_{i}^{\mathrm{eq}}\left(\rho, \mathbf{u}_{\mathrm{s}}\right)
$$

154 where $\mathbf{u}_{\mathrm{s}}$ is the velocity of the solid phase, whose value is set as 0 for the constrained melting. Note that the density in Eq. (9)(9) should be first calculated using Eq. (10)(10) to ensure the local mass conservation. Here, for liquid phase $\left(f_{l}=1\right)$, Eqs. (1)(1), (9)(9) and $(10)(10)$ become a standard LB model, while for the solid phase $\left(f_{l}=0\right)$, Eq. (9) $(9)$ become $f_{\mathrm{i}}=f_{\mathrm{i}}^{\text {eq }}\left(\rho, \mathbf{u}_{\mathrm{s}}\right)$, which means

158 that the nonslip velocity $u=u_{\mathrm{s}}$ can be strictly satisfied. In other words, there is no nonphysical flow in the solid phase. The macroscopic quantities are calculated as follows. 
$\rho=\sum_{i} f_{i}^{*}-\frac{\Delta t}{2} \frac{\rho u_{r}}{r}, \quad \mathbf{u}=\left(\sum_{i} \mathbf{e}_{i} f_{i}+\frac{\Delta t}{2} \mathbf{F}\right) /\left(\sum_{i} f_{i}^{*}\right)$

where $\mathbf{e}_{i}$ is the discrete velocity and given in Eq. (11).

$\mathbf{e}_{i}= \begin{cases}c(0,0)^{\mathrm{T}} & i=0 \\ c(\cos [(i-1) \pi / 2], \sin [(i-1) \pi / 2])^{\mathrm{T}} & i=1-4 \\ \sqrt{2} c(\cos [(2 i-1) \pi / 4], \sin [(2 i-1) \pi / 4])^{\mathrm{T}} & i=5-8\end{cases}$

\subsubsection{Axisymmetric thermal LB model}

The total enthalpy distribution function $g(\mathrm{x}, \mathrm{t})$ of the axisymmetric LB model, which used for the temperature field, can be expressed as Eq. (12).

$g_{i}(r+\Delta r, t+\Delta t)=g_{i}(r, t)-\omega_{g}\left[g_{i}(r, t)-g_{i}^{\mathrm{eq}}(r, t)\right]$

where the factor $\omega_{g}$ and the equilibrium distribution function $g^{\text {eq }}$ are shown as follows [23, 29].

$\omega_{g}=\left(1+e_{i r} \frac{3 \alpha}{r c^{2}}\right) /\left(0.5+\frac{3 \alpha}{\Delta t c^{2}}\right)$
$g^{\mathrm{eq}}= \begin{cases}H-C_{\mathrm{p}, \mathrm{ref}} T+w_{i} C_{\mathrm{p}, \mathrm{ref}} T-w_{i} C_{\mathrm{p}} T \frac{u^{2}}{2 c_{\mathrm{s}}^{2}} & i=0 \\ w_{i} C_{\mathrm{p}, \mathrm{ref}} T+w_{i} C_{\mathrm{p}} T\left[\frac{\left(\mathbf{e}_{i} \cdot \mathbf{u}\right)}{c_{\mathrm{s}}^{2}}+\frac{\left(\mathbf{e}_{i} \cdot \mathbf{u}\right)^{2}}{2 c_{\mathrm{s}}^{4}}-\frac{u^{2}}{2 c_{\mathrm{s}}^{2}}\right] & i=1-8\end{cases}$

where $C_{\mathrm{p}, \text { ref }}$ is a reference specific heat, which was used to make the specific heat $C_{\mathrm{p}}$ and thermal conductivity $\lambda$ of the PCM decoupled [46], $w_{\mathrm{i}}$ is the weight coefficient, which equals to $4 / 9$ when $i=0$, and $1 / 9$ for $i=1-4$, as well as $1 / 36$ for $i=5-8, C_{\mathrm{p}}$ and $\alpha$ are different in the liquid domain and solid one, as defined as follows.

$\alpha=f_{l} \alpha_{l}+\left(1-f_{l}\right) \alpha_{\mathrm{s}}$

$C_{\mathrm{p}}=f_{l} C_{\mathrm{p}, l}+\left(1-f_{l}\right) C_{\mathrm{p}, \mathrm{s}}$

$C_{\mathrm{p}, \text { ref }}=2 C_{\mathrm{p}, l} C_{\mathrm{p}, \mathrm{s}} /\left(C_{\mathrm{p}, l}+C_{\mathrm{p}, \mathrm{s}}\right)$ 
$\alpha=\frac{\lambda}{\rho c}$

171 The macroscopic variable, total enthalpy $H$, is calculated by Eq. (19).

$H=\sum_{i} g_{i}$

The volume fraction of the liquid $f_{l}$ and temperature $T$ can be determined by the thermodynamical relations as descrbied in Eq. $(20)(20)$ and Eq. $(21)(21)$. Here, $f_{l}$ is a local variable, representing the liquid fraction in the control cell of a lattice node, $f_{l}=0$ and $f_{l}=1$ mean that the lattice node is the solid part and liquid part, respectively. The value of $f_{l}$ between 0 and 1 represents the part that phase change happens.

$$
\begin{gathered}
f_{l}= \begin{cases}0 & H \leq H_{\mathrm{s}} \\
\left(H-H_{\mathrm{s}}\right) /\left(H_{l}-H_{\mathrm{s}}\right) & H_{\mathrm{s}}<H<H_{l} \\
1 & H \geq H_{l}\end{cases} \\
T= \begin{cases}H / C_{\mathrm{p}, \mathrm{s}} & H \leq H_{\mathrm{s}} \\
H_{\mathrm{s}}+\frac{H-H_{\mathrm{s}}}{H_{l}-H_{\mathrm{s}}}\left(T_{l}-T_{\mathrm{s}}\right) & H_{\mathrm{s}}<H<H_{l} \\
T_{l}+\left(H-H_{l}\right) / C_{\mathrm{p}, l} & H \geq H_{l}\end{cases}
\end{gathered}
$$
temperature $T_{l}\left(T_{l} \geq T_{\mathrm{s}}\right)$, respectively. The equal symbol means that the phase change process

\section{$180 \quad$ 2.2.4 Model validation}

181 In order to validate the enthalpy-based axisymmetric LB model, a baseline case of the melting process in an annulus between two coaxial vertical cylinders was tested [29], see Fig. 2Fig. 2.

183 The radii of the inner and outer cylinders are $r_{0}$ and $2 r_{0}$, while the height $l_{\mathrm{z}}$ equals to $2 r_{0}$. Initially,

184 the annulus was filled with a solid PCM at the melting temperature $T_{\mathrm{m}}$. Then, the wall 

defined by Eq. (22) $(22)$.

$$
N u=\frac{\int_{0}^{l_{z}} q_{r} d y}{\lambda_{l}\left(T_{\mathrm{b}}-T_{\mathrm{m}}\right)}, \quad F o=\frac{\alpha t}{l_{z}^{2}}
$$
respectively.

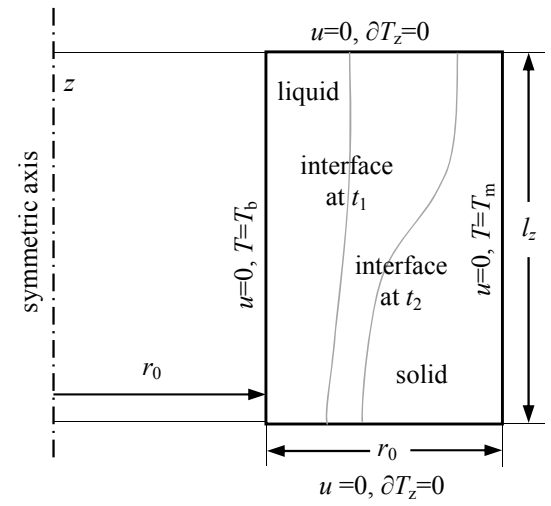

liquid fraction, and the steady temperature field and flow field results were compared with the results of a published literature [29], see Fig. 3Fig. 3. It can be seen that those simulation results

199 were very close to the published results. The maximum errors of $N u$ number and global liquid

200 fraction was $3.50 \%$ and $3.06 \%$, respectively. This indicated that the enthalpy-based axisymmetric

201 LB model can predict the solid-liquid phase change heat transfer accurately. 

1.0. The detailed thermophysical properties of the PCM used were listed in Table 1Table 1. The

210 viscosity in the lattice space was chosen as $2.0 \times 10^{-2}$, and the thermal diffusivity was $3.35 \times 10^{-4}$ in

$$
\operatorname{Pr}=\frac{v}{\alpha}
$$

$$
\mathbf{M}=\left(\begin{array}{ccccccccc}
+1 & +1 & +1 & +1 & +1 & +1 & +1 & +1 & +1 \\
-4 & -1 & -1 & -1 & -1 & +2 & +2 & +2 & +2 \\
0 & -2 & -2 & -2 & -2 & +1 & +1 & +1 & +1 \\
0 & +1 & 0 & -1 & 0 & +1 & -1 & -1 & +1 \\
0 & -2 & 0 & +2 & 0 & +1 & -1 & -1 & +1 \\
0 & 0 & +1 & 0 & -1 & +1 & +1 & -1 & -1 \\
0 & 0 & -2 & 0 & +2 & +1 & +1 & -1 & -1 \\
0 & +1 & -1 & +1 & -1 & 0 & 0 & 0 & 0 \\
0 & 0 & 0 & 0 & 0 & +1 & -1 & +1 & -1
\end{array}\right)
$$

214 tested to make sure that the solution was independent from the adopted grid density, see Fig.4. 


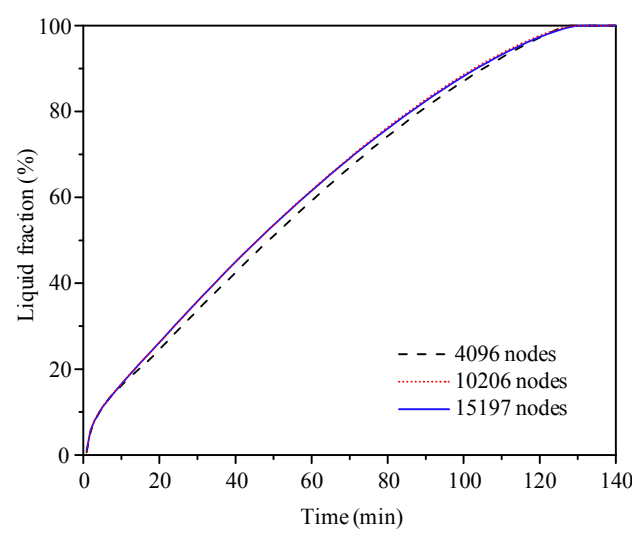

Fig. 4 Variations of the global liquid fraction vs. time at different grid sizes.

\section{Results and discussions}

\subsection{Comparison of numerical and experimental results} qualitative observation of the PCM melting was discussed. The experimental photographs of the constrained melting were shown in Fig. 5Fig. 5, while the simulation results were presented in left half of each circle, whereas the streamlines were drawn on the right half. Then, these observations were linked to the quantitative results of LBM simulations and detailed 
232

233

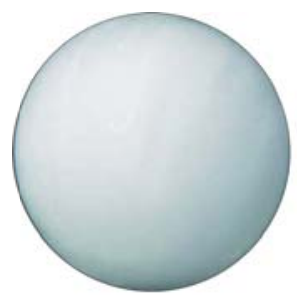

(a) $0 \mathrm{~min}$

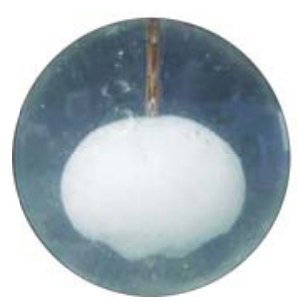

(e) $80 \mathrm{~min}$

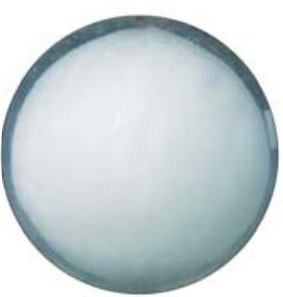

(b) $20 \mathrm{~min}$

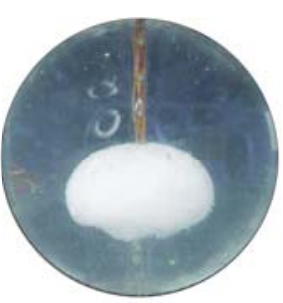

(f) $100 \mathrm{~min}$

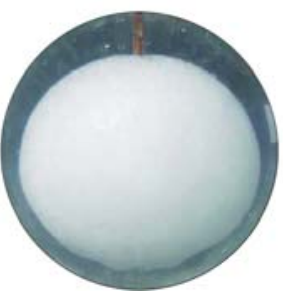

(c) $40 \mathrm{~min}$

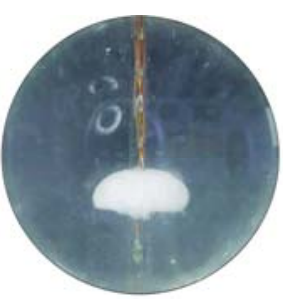

(g) $120 \mathrm{~min}$

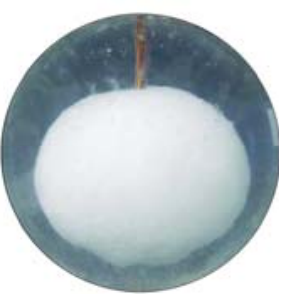

(d) $60 \mathrm{~min}$

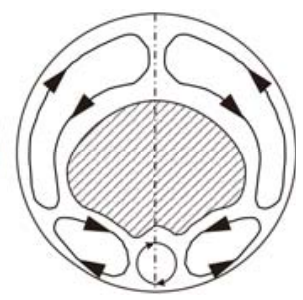

(h) schematic diagram

Fig. 5 Instantaneous photographs of the spherical capsule during the constrained melting $[19,20]$.

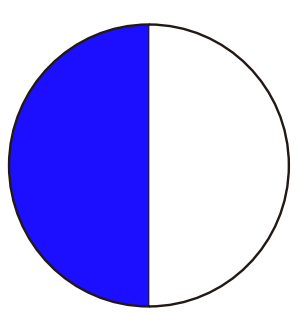

(a) $0 \mathrm{~min}$

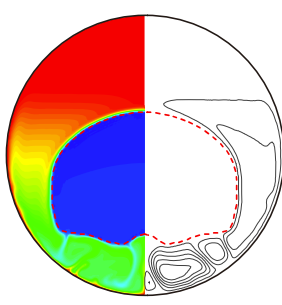

(e) $80 \mathrm{~min}$

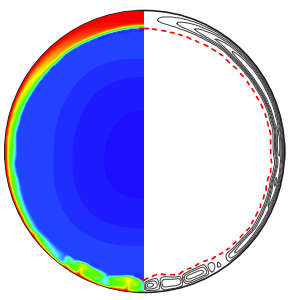

(b) $20 \mathrm{~min}$

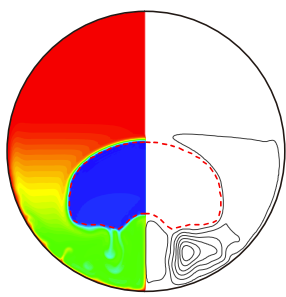

(f) $100 \mathrm{~min}$

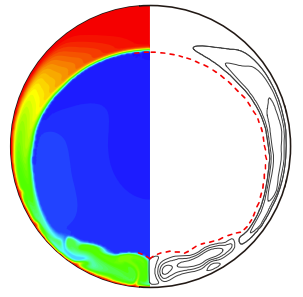

(c) $40 \mathrm{~min}$

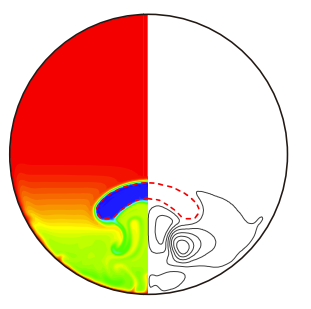

(g) $120 \mathrm{~min}$

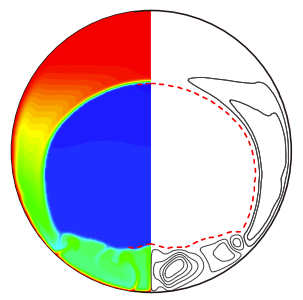

(d) $60 \mathrm{~min}$

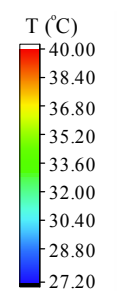

Formatted: Font: $10.5 \mathrm{pt}$ Formatted: Font: $10.5 \mathrm{pt}$ 
LBM simulation results agreed well with the experimental data obtained from Tan $[19,20]$. At

244 the beginning, a thin layer of the liquid between the solid PCM and the spherical wall was formed, while the solid PCM remained a perfect sphere, as shown in Fig. 5Fig. 5(b) and Fig. 6Fig. 6(b). This is because the dominated factor of the PCM melting was the conduction in this stage. As time elapsed, a liquid layer was developed and thus the heat conduction was significantly reduced. An oval shape phase front was formed at the top of the solid PCM, while a waviness of the surface was observed at the bottom of the solid PCM, as illustrated in Fig. 5Fig. 5(c)-(f) and Fig. 6Fig. 6(c)-(f). This could be attributed to the natural convection, which occurred as a result of the warm liquid PCM rose along the hot spherical wall while the cool liquid PCM flowed down around the solid PCM. At the upper part of the spherical capsule, the "hot" spherical wall was higher than the "cold" solid PCM, which formed a steady thermal convection cell. On the contrary, the "hot" wall was lower than the "cold" solid PCM at the bottom part of the capsule. It would cause an intense natural convection and formed several chaotic convection cells until the solid PCM was melted completely, which further made the bottom of the solid PCM to be a waviness shape. Thus, a very interesting phenomenon can be observed in this stage that the thermal stable and unstable layers were co-existed in the spherical capsule. The horizontal stratified fluid layers can be observed in thermal stable layer, while there were several recirculating cells in the unstable layer. At the $120^{\text {th }}$ minute, the position of the solid PCM was

261 lower than the center of the sphere, thus clearly pointing to the preferential melting in the top top and bottom concave-down surfaces. 


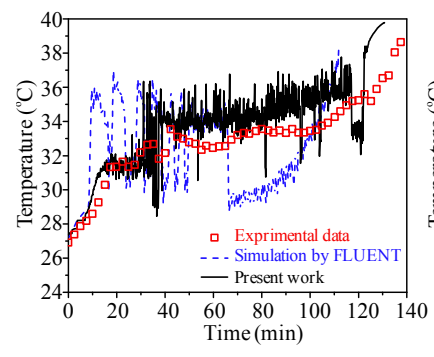

(a) Point A

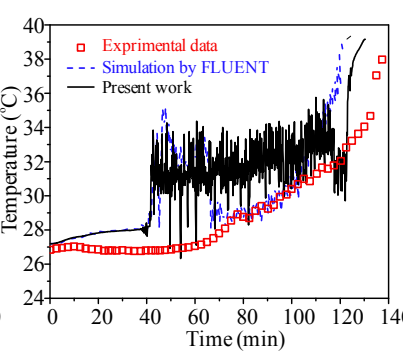

(b) Point B

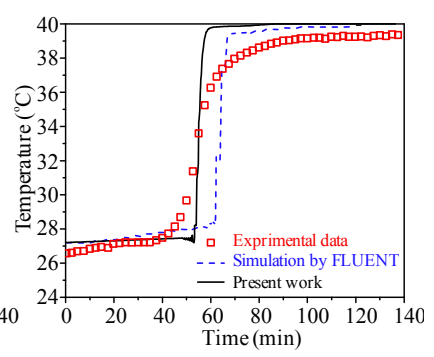

(c) Point G

Fig. 7 Comparison of the simulated and experimental results $[19,21]$ at different typical points (A, B, G).

Table 2 Comparison of the results between the use of conventional CFD method and LBM

\begin{tabular}{cccccc}
\hline \multirow{2}{*}{ Model } & \multirow{2}{*}{ Software } & Computed times & \multicolumn{3}{c}{ Errors (\%) } \\
& & (Parallel number) & Location & Max & Average \\
\hline \multirow{2}{*}{ Conventional } & \multirow{2}{*}{ FLUENT } & $225 \min (1)$ & A & 23.92 & 7.92 \\
CFD & & $178 \min (4)$ & B & 29.32 & 7.93 \\
& & G & 21.61 & 3.18 \\
\multirow{3}{*}{ LBM } & Visual studio & $212 \min (1)$ & A & 11.09 & 4.23 \\
& (Fortran) & $148 \min (4)$ & B & 17.89 & 8.26 \\
& & & G & 12.79 & 2.81 \\
\hline
\end{tabular}

269 CFD simulations [21] and experimental data [19] at three measure points (A, B and G). The

270 results showed that $\mathrm{LBM}$ had much higher parallelism than CFD, resulting in a higher efficiency

271 of using LBM than that using CFD when the parallel number was more than two. Besides, the

272 LBM results were much closer to the experimental data than the results obtained using the

273 conventional CFD method [21]. For example, compared with the conventional CFD results at

274 point $\mathrm{A}$, the maximum and average errors of the LBM results can be reduced from $23.92 \%$ and $275 \mid 7.92 \%$ to $11.09 \%$ and $4.23 \%$, respectively. From Fig. 7 Fig. 7 (a) and (b), the disordered

276 temperature data at the points A and B reflected the chaotic convection motion in the unstable

277 layer at the bottom of the sphere. The chaotic temperature readings would be observed until there

278 was no more solid PCM left in the capsule. From Fig. 7Fig. 7(c), the computed temperatures at 
point $\mathrm{G}$ were in a good agreement with the experimental data. The temperature curves were quite smooth, which was due to the stable nature of the liquid layer at the upper part of the sphere. It reflected that there was no strong convective motion existed, and confirmed that these points were located in the thermal stable layer. Similar figures were obtained at the points D, E, F and H,

\subsection{Effects of non-uniform properties of the PCM}

where $T_{\text {surf }}$ and $T_{\mathrm{m}}$ were the surface temperature of the spherical capsule and the melting temperature of the PCM, respectively, $l_{z}$ was the character length, which can be set as the diameter, $g$ was the acceleration of gravity, $L$ was the latent heat of fusion, and $\beta$ was the volume expansivity.

So far, there is insufficient studies focusing on the differences of the thermal diffusivity between the liquid and solid PCMs in existing non-dimensional parameters. In general, the thermal diffusivity of the solid PCM is larger than of the liquid state. It is a key parameter to the heat transfer process, and thus further affects the phase change process inside the capsule. In this study, a non-dimensional parameter $\left(\alpha_{\mathrm{s}} / \alpha_{l}\right)$, which represented the thermal diffusivity ratio of the solid PCM to the liquid PCM, was used to investigate the effect of non-uniform properties of the 
PCM on the melting process. In order to observe an obvious regularity, the initial melting temperature in this section was set as $18.2^{\circ} \mathrm{C}$ (i.e. $10^{\circ} \mathrm{C}$ below the melting temperature). Here, the melting state was described by the global liquid fraction. Fig. 8Fig. 8 shows the variation in the global liquid fraction as a function of the time at different thermal diffusivity ratios $\left(\alpha_{\mathrm{s}} / \alpha_{l}\right)$. Based on the experiments [47], the thermal diffusivity ratio of paraffin varied from 1.1 to 1.7 , and thus are shown in Fig. 9Fig. 9 (a) and (b) respectively. Fig. 9Fig. 9 (a) could be used to analyze the PCM melting behavior, while Fig. 9Fig. 9 (b) could be helpful when examining the magnitude of decreased with the increase of thermal diffusivity ratio.

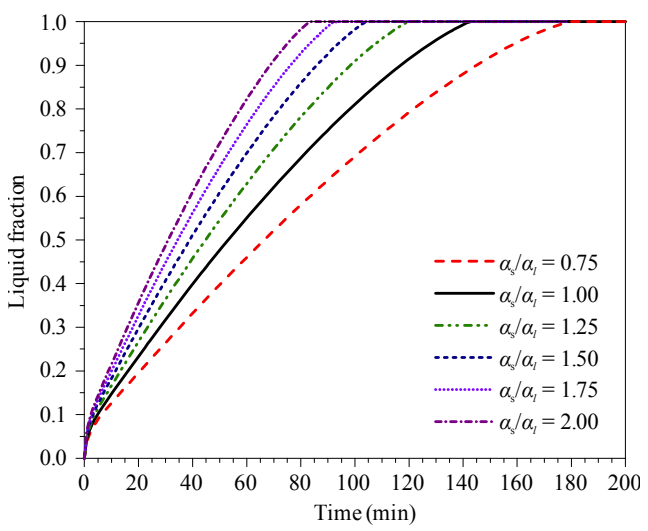

Fig. 8 Variations in the global liquid fraction versus time at different thermal diffusivity ratios.

In order to further investigate the effect of the thermal diffusivity ratio on the melting rate, the variation in the melting rate as a function of the time and global liquid fraction at different ratios the melting rate under the same melting state (approximately expressed by the global liquid 
fraction). It can be easily seen that there existed a rapid decrease in the melting rate at the

beginning, which was due to the fact that the effect of the heat conduction was significantly

reduced as the liquid layer progressed, as mentioned in Section 3.2. Then, the effect of the

natural convection gradually became the dominated factor of the PCM melting, and thus the

melting rate would fluctuate apparently because of the chaotic convection motion (as mentioned

in Section 3.3). The transformation of the dominant factor from conduction to convection 9Fig. 9). weakened. The double increase in the thermal diffusivity ratio resulted in the melting rate increased by $64 \%$ when the global liquid fraction was about 0.13 . As the global liquid fraction increases, this influence was somewhat enhanced: the melting rate was improved from 1.64 to

1.79 times when the thermal diffusivity ratio was expected to be doubled.

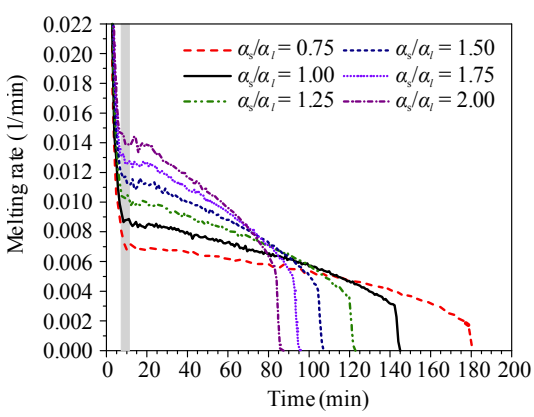

(a) Melting rate versus time

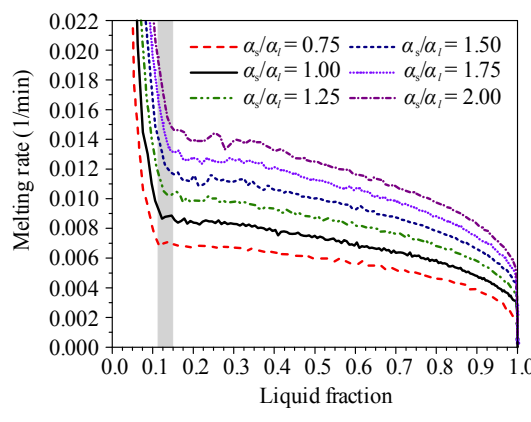

(b) Melting rate versus global liquid fraction
Formatted: Font: $10.5 \mathrm{pt}$

Formatted: Font: $10.5 \mathrm{pt}$ 


\section{$335 \quad 3.3$ Effects of the surface temperature and capsule size}

In this section, the effects of the capsule size and the surface temperature on the melting

342 different surface temperatures were expressed by the Ste number. It was the ratio of the sensible heat to the latent heat of the PCM and was defined by Eq. (25)(25).

The simulation was performed at three different Ste numbers and six different capsule diameters, namely Ste $=0.08059\left(T_{\text {surf }}=35^{\circ} \mathrm{C}\right)$, Ste $=0.13225\left(T_{\text {surf }}=40^{\circ} \mathrm{C}\right), S t e=0.18392\left(T_{\text {surf }}=45^{\circ} \mathrm{C}\right)$ and $D_{1}=101.66 \mathrm{~mm}, D_{2}=50.83 \mathrm{~mm}, D_{3}=25.42 \mathrm{~mm}, D_{4}=1.017 \mathrm{~mm}, D_{5}=508.3 \mu \mathrm{m}, D_{6}=254.2 \mu \mathrm{m}$, respectively. The first three spherical capsules $\left(D_{1}-D_{3}\right)$ were in the macro-scale, while the others were in the micro-scale $[15,16]$. Fig. 10Fig. 10 shows the variations in the global liquid fraction versus the non-dimensional time $(F o)$ at different Ste numbers and capsule sizes. The slopes of these graphs represented the melting rate. The highest melting rate occurred at the beginning of the melting process because of the heat conduction. For each capsule size, the global liquid fraction increased more rapidly with a larger surface temperature (or Ste), whereas the time required for completing the melting reduced with the increase of Ste. The effects of these parameters on the melting time and melting rate were different at macro- and micro- scales. In order to clearly illustrate the melting times under different conditions, the detailed information 


\begin{tabular}{cccccc}
\hline \multirow{2}{*}{ Diameter $(\mathrm{mm})$} & $\begin{array}{c}T_{\text {Surf }}\left({ }^{\circ} \mathrm{C}\right) \\
\text { Ste }\end{array}$ & $\begin{array}{c}\text { Time }(\mathrm{min}) \\
\text { Fo }\end{array}$ & Diameter $(\mu \mathrm{m})$ & $\begin{array}{c}T_{\text {Surf }}\left({ }^{\circ} \mathrm{C}\right) \\
\text { Ste }\end{array}$ & $\begin{array}{c}\text { Time }(\mathrm{s}) \\
\text { 101.66 }\end{array}$ \\
\hline \multirow{5}{*}{50.83} & $35(0.08059)$ & $274(0.1331)$ & & $35(0.08059)$ & $7.92(0.6403)$ \\
& $40(0.13225)$ & $129(0.0625)$ & \multirow{2}{*}{1017.0} & $40(0.13225)$ & $4.56(0.3689)$ \\
& $45(0.18392)$ & $76(0.0371)$ & & $45(0.18392)$ & $3.20(0.2589)$ \\
& $35(0.08059)$ & $97(0.1878)$ & & $35(0.08059)$ & $1.98(0.6404)$ \\
& $40(0.13225)$ & $51(0.0987)$ & \multirow{2}{*}{508.3} & $40(0.13225)$ & $1.13(0.3690)$ \\
& $45(0.18392)$ & $33(0.0634)$ & & $45(0.18392)$ & $0.80(0.2590)$ \\
& $35(0.08059)$ & $39(0.3002)$ & & $35(0.08059)$ & $0.49(0.6404)$ \\
& $40(0.13225)$ & $20(0.1557)$ & 254.2 & $40(0.13225)$ & $0.29(0.3690)$ \\
& $45(0.18392)$ & $13(0.1009)$ & & $45(0.18392)$ & $0.20(0.2592)$ \\
\hline
\end{tabular}

Fig.10 Variations in the global liquid fraction versus Fo at different Ste numbers and capsule sizes.

Table 3 The end time of the melting process at different Ste numbers and capsule sizes

It was interesting to note that for each surface temperature (or Ste), the actual melting time of a micro-capsule $\left(D_{4}-D_{6}\right)$ was always less than that of a macro-capsule $\left(D_{1}-D_{3}\right)$, while the non-dimensional form of the fully melting time showed an opposite trend. The former was mainly because the heat inputs to the capsule and PCM content were not the same when the surface temperature of the capsule maintained as constant, and thus the fully melting time was

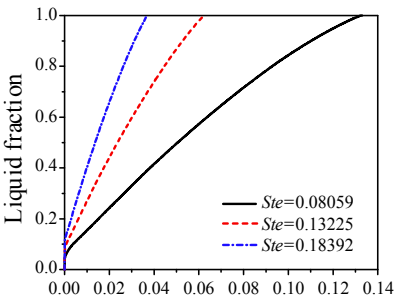

(a) $D_{1}=101.66 \mathrm{~mm}$

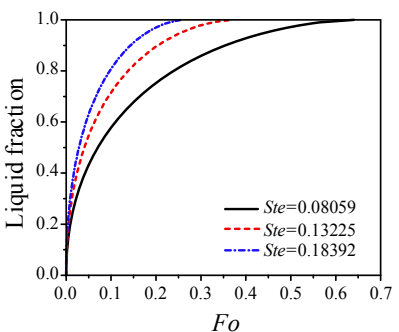

(d) $D_{4}=1016.6 \mu \mathrm{m}$

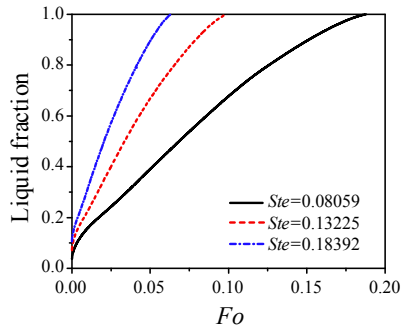

(b) $D_{2}=50.83 \mathrm{~mm}$

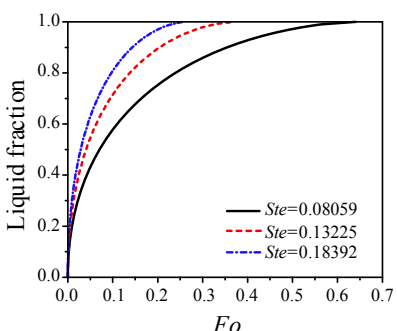

(e) $D_{5}=508.3 \mu \mathrm{m}$

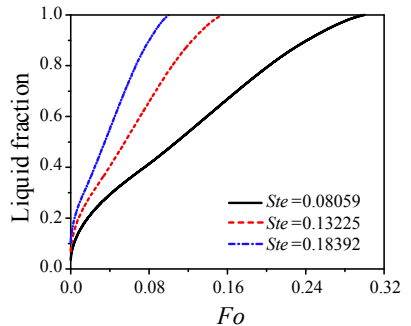

(c) $D_{3}=25.42 \mathrm{~mm}$

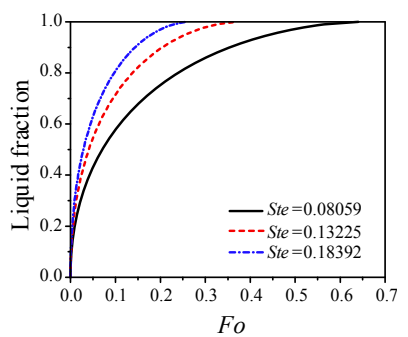

(f) $D_{6}=254.2 \mu \mathrm{m}$ 

near-invariable fully melting time. In addition, there was an interesting phenomenon in Fig.10Fig.10 that the variation in the global liquid fraction was nearly linear at the macro-scale while a parabolic trend at the micro-scale was observed. It may be caused by the fact that the convection played a more important role than the conduction during the most of the melting

always decreased with the decrease of the capsule size. The latter was because the convection effect was decreased with the decrease of the capsule size. During the melting process, the liquid PCM increased and thus the heat conduction was significantly reduced, which caused a long fully melting time. In the following discussion, the fully melting time was expressed by $F_{O}$ instead of the actual time.

At the macro-scale, based on the data presented in Fig.10Fig. 10(a)-(c) and Table 3Fable 3, it can be extracted that $28.6 \%$ increase in the surface temperature was from $35^{\circ} \mathrm{C}$ to $45^{\circ} \mathrm{C}$ resulted in about $66.3-72.1 \%$ decrease in the melting time for each constant diameter, respectively. The effect of the surface temperature on the melting time slightly increased with the increase of the diameter of the capsule. For example, when the surface temperature was $45^{\circ} \mathrm{C}$, the increase of the capsule diameter varied from $25.42 \mathrm{~mm}$ to $50.83 \mathrm{~mm}$ and from $50.83 \mathrm{~mm}$ to $101.66 \mathrm{~mm}$ resulted in $37.2-41.4 \%$ decrease in the melting time, respectively. The surface temperature had a more prominent effect on the melting time than the capsule size.

At the micro-scale, as shown in Fig.10Fig. 10(d)-(f) and Table 3Fable 3, the increase of the surface temperature varied from $35^{\circ} \mathrm{C}$ to $45^{\circ} \mathrm{C}$, resulted in about $59.6 \%$ decrease in the fully melting time for each constant diameter, respectively. However, when the size of the capsule increased from $254.2 \mu \mathrm{m}$ to $508.3 \mu \mathrm{m}$, or from $508.3 \mu \mathrm{m}$ to $1.017 \mathrm{~mm}$, there was a process at the macro-scale, while the conduction was dominated at the micro-scale.
Formatted: Font: $10.5 \mathrm{pt}$ Formatted: Font: $10.5 \mathrm{pt}$ 


\section{Conclusions}

Flow and heat transfer evolution of PCMs inside an encapsulated capsule due to natural convection melting was simulated using an enthalpy-based axisymmetric lattice Boltzmann model (LBM). The simulation results matched well with the experimental data, which presented that the thermal stable and unstable layers were coexisted in the spherical capsule during the melting process. Moreover, the results of LBM presented a desirable accuracy and efficiency compared to that of the traditional CFD methods in this situation.

In order to study the effect of the difference between the liquid thermal diffusivity and solid thermal diffusivity on the melting process, a dimensionless parameter called solid/liquid thermal diffusivity ratio was introduced. The fully melting time decreased by $40.6 \%$ (e.g. from $143 \mathrm{~min}$ to $85 \mathrm{~min}$ ) when increasing the thermal diffusivity ratio from 1.0 to 2.0. During the melting process, however, the effect of the thermal diffusivity ratio on the melting rate was different. With continuous increase of the thermal diffusivity ratio, the melting rate enhancement was decreased rapidly with the progression of the melting process when the global liquid fraction was less than 0.13 , and then the melting rate enhancement increased slightly from 1.64 to 1.79 times until the melting process was completed.

Finally, the effects of the surface temperature and capsule sizes on the melting time and melting rate were investigated at different scales by a set of dimensionless numbers, such as Ste and Fo. The surface temperature had a more significant effect than the spherical capsule size on the fully melting time. In addition, the effect of the capsule sizes on the melting process was different. At the macro-scale (i.e. diameters from $1 \mathrm{~mm}$ or greater than $1 \mathrm{~cm}$ ), the double increase in the diameter of a capsule resulted in the fully melting time reduced by $35.8-37.3 \%$; while at 
412 the micro-scale (i.e. diameters from $1 \mu \mathrm{m}$ to $1 \mathrm{~mm}$ ), there was a near-invariable fully melting

413 time when the capsule diameter was doubled.

\section{Acknowledgements}

415 This work was supported by National Natural Science Foundation of China with Grant numbers

416 of 51678102 and 51508067.

\section{References}

418 [1] Z.J. Ma, W.Y. Lin, M.I. Sohel, Nano-enhanced phase change materials for improved building

419 performance, Renewable and Sustainable Energy Reviews, 58 (2016) 1256-1268.

420

421

[2] A. Pasupathy, R. Velraj, R.V. Seeniraj, Phase change material-based building architecture for thermal management in residential and commercial establishments, Renewable and Sustainable Energy Reviews, 12 (2008) 39-64.

[3] F. Kuznik, D. David, K. Johannes, J.-J. Roux, A review on phase change materials integrated in building walls, Renewable and Sustainable Energy Reviews, 15 (2011) 379-391.

[4] W.Y. Lin, Z.J. Ma, Using Taguchi-Fibonacci search method to optimize phase change materials enhanced buildings with integrated solar photovoltaic thermal collectors, Energy, 106 (2016) 23-37.

[5] R. Kandasamy, X.Q. Wang, A.S. Mujumdar, Application of phase change materials in thermal management of electronics, Applied Thermal Engineering, 27 (2007) 2822-2832.

[6] T.J. Lu, Thermal management of high power electronics with phase change cooling, International Journal of Heat and Mass Transfer, 43 (2000) 2245-2256. 
[7] N.S. Bondareva, M.A. Sheremet, Flow and heat transfer evolution of PCM due to natural convection melting in a square cavity with a local heater, International Journal of Mechanical Sciences, 134 (2017) 610-619.

[8] W.Y. Lin, Z.J. Ma, P. Cooper, M.I. Sohel, L.W. Yang, Thermal performance investigation and optimization of buildings with integrated phase change materials and solar photovoltaic thermal collectors, Energy and Buildings, 116 (2016) 562-573.

[9] H.S. Xue, Experimental investigation of a domestic solar water heater with solar collector coupled phase-change energy storage, Renewable Energy, 86 (2016) 257-261.

[10] A. Shukla, D. Buddhi, R.L. Sawhney, Solar water heaters with phase change material thermal energy storage medium: a review, Renewable and Sustainable Energy Reviews, 13 (2009) 2119-2125.

[11] H.B. Li, Z.J. Peng, B.Q. Ma, P.L. Wang, J.Q. Li, Numerical analysis of thermal energy charging performance of spherical Cu@Cr@Ni phase-change capsules for recovering high-temperature waste heat, Journal of Materials Research, 32 (2017) 1138-1148.

[12] S. Zhang, J. Niu, Experimental investigation of effects of supercooling on microencapsulated phase-change material (MPCM) slurry thermal storage capacities, Solar Energy Materials and Solar Cells, 94 (2010) 1038-1048.

[13] N.S. Roberts, R. Al-Shannaq, J. Kurdi, S.A. Al-Muhtaseb, M.M. Farid, Efficacy of using slurry of metal-coated microencapsulated PCM for cooling in a micro-channel heat exchanger, Applied Thermal Engineering, 122 (2017) 11-18.

[14] M. Kong, J.L. Alvarado, C. Thies, S. Morefield, C.P. Marsh, Field evaluation of microencapsulated phase change material slurry in ground source heat pump systems, Energy, 
[15] R. Jacob, F. Bruno, Review on shell materials used in the encapsulation of phase change materials for high temperature thermal energy storage, Renewable and Sustainable Energy Reviews, 48 (2015) 79-87.

[16] M.A. Augustin, L. Sanguansri, Microencapsulation technologies, in: Y.H. Roos, Y.D. Livney NY, 2017, pp. 119-142.

[17] M. Jurkowska, I. Szczygieł, Review on properties of microencapsulated phase change materials slurries (mPCMS), Applied Thermal Engineering, 98 (2016) 365-373.

[18] J.M. Khodadadi, Y. Zhang, Effects of buoyancy-driven convection on melting within spherical containers, International Journal of Heat and Mass Transfer, 44 (2001) 1605-1618.

[19] F.L. Tan, Constrained and unconstrained melting inside a sphere, International Communications in Heat and Mass Transfer, 35 (2008) 466-475.

[20] F.L. Tan, S.F. Hosseinizadeh, J.M. Khodadadi, L. Fan, Experimental and computational study of constrained melting of phase change materials (PCM) inside a spherical capsule, International Journal of Heat and Mass Transfer, 52 (2009) 3464-3472.

[21] H. Sattari, A. Mohebbi, M.M. Afsahi, A. Azimi Yancheshme, CFD simulation of melting process of phase change materials (PCMs) in a spherical capsule, International Journal of Refrigeration, 73 (2017) 209-218

[22] M. Ghalambaz, A. Doostani, A.J. Chamkha, M.A. Ismael, Melting of nanoparticles-enhanced phase-change materials in an enclosure: Effect of hybrid nanoparticles, International Journal of Mechanical Sciences, 134 (2017) 85-97. 
[23] R.Z. Huang, H.Y. Wu, P. Cheng, A new lattice Boltzmann model for solid-liquid phase change, International Journal of Heat and Mass Transfer, 59 (2013) 295-301.

[24] R.Z. Huang, H.Y. Wu, Total enthalpy-based lattice Boltzmann method with adaptive mesh refinement for solid-liquid phase change, Journal of Computational Physics, 315 (2016) 65-83.

[25] M. Jourabian, M. Farhadi, A.A.R. Darzi, Simulation of natural convection melting in an inclined cavity using lattice Boltzmann method, Scientia Iranica, 19 (2012) 1066-1073.

[26] W.B. Zhu, M. Wang, H. Chen, 2D and 3D lattice Boltzmann simulation for natural convection melting, International Journal of Thermal Sciences, 117 (2017) 239-250.

[27] F.J. Yao, K. Luo, H.L. Yi, M. Xie, Analysis of melting with natural convection and volumetric radiation using lattice Boltzmann method, International Journal of Heat and Mass Transfer, 112 (2017) 413-426.

[28] M. Jourabian, M. Farhadi, Melting of nanoparticles-enhanced phase change material (NEPCM) in vertical semicircle enclosure: numerical study, Journal of Mechanical Science and Technology, 29 (2015) 3819-3830.

[29] D. Li, Q.1. Ren, Z.X. Tong, Y.L. He, Lattice Boltzmann models for axisymmetric solid-liquid phase change, International Journal of Heat and Mass Transfer, 112 (2017) 795-804.

[30] M. Jourabian, M. Farhadi, A.A.R. Darzi, Accelerated melting of PCM in a multitube annulus-type thermal storage unit using lattice Boltzmann simulation, Heat Transfer-Asian Research, 4 (2017) 1-27.

[31] K. Luo, F.J. Yao, H.L. Yi, H.P. Tan, Lattice Boltzmann simulation of convection melting in complex heat storage systems filled with phase change materials, Applied Thermal Engineering, 86 (2015) 238-250. 
[32] H. Inaba, New challenge in advanced thermal energy transportation using functionally thermal fluids, International Journal of Thermal Sciences, 39 (2000) 991-1003.

[33] P. Charunyakorn, S. Sengupta, S.K. Roy, Forced convection heat transfer in microencapsulated phase change material slurries: flow in circular ducts, International Journal of Heat and Mass Transfer, 34 (1991) 819-833

[34] Y.P. Zhang, X.X. Hu, Q. Hao, X. Wang, Convective heat transfer enhancement of laminar flow of latent functionally thermal fluid in a circular tube with constant heat flux: internal heat source model and its application, Science in China Series E: Technological Sciences, 46 (2003) 131-140.

[35] Y.L. Hao, Y.X. Tao, A numerical model for phase-change suspension flow in microchannels, Numerical Heat Transfer, Part A: Applications, 46 (2004) 55-77.

[36] K.Q. Xing, Y.X. Tao, Y.L. Hao, Performance evaluation of liquid flow with PCM particles in microchannels, Journal of Heat Transfer, 127 (2005) 931-940.

[37] P. Zhang, X.J. Shi, Thermo-fluidic characteristics of ice slurry in horizontal circular pipes, International Journal of Heat and Mass Transfer, 89 (2015) 950-963.

[38] L.K. Liu, G. Alva, Y.T. Jia, X. Huang, G.Y. Fang, Dynamic thermal characteristics analysis of microencapsulated phase change suspensions flowing through rectangular mini-channels for thermal energy storage, Energy and Buildings, 134 (2017) 37-51.

[39] C.J. Ho, A continuum model for transport phenomena in convective flow of solid-liquid phase change material suspensions, Applied Mathematical Modelling, 29 (2005) 805-817.

[40] J.H. Li, W.1. Huang, Toward Mesoscience - The Principle of Compromise in Competition, Springer Berlin Heidelberg, Berlin, 2014. 
[41] J.H. Li, Approaching virtual process engineering with exploring mesoscience, Chemical Engineering Journal, In Press, Corrected Proof (2014).

[42] Q. Lin, S.G. Wang, J.H. Wang, S.L. Song, Numerical simulation of constrained melting inside a spherical capsule by lattice Boltzmann method (On-line first), CIESC Journal, (2018) In Publish.

[43] Q. Li, Y.L. He, G.H. Tang, W.Q. Tao, Improved axisymmetric lattice Boltzmann scheme, Physical Review E, 81 (2010) 056707.

[44] A.A. Mohamad, Lattice Boltzmann method: fundamentals and engineering applications with computer codes, Springer Science \& Business Media, Dordrecht, Netherlands, 2011.

[45] Q. Liu, Y.L. He, An enthalpy-based multiple-relaxation-time lattice Boltzmann method for solid-liquid phase change heat transfer in metal foams, arXiv preprint arXiv:1701.00702, (2016) $1-40$

[46] R.Z. Huang, H.Y. Wu, Phase interface effects in the total enthalpy-based lattice Boltzmann model for solid-liquid phase change, Journal of Computational Physics, 294 (2015) 346-362.

[47] J.H. Zhang, X.Q. Xie, Y. Zhou, Q.L. Li, Experimental research on thermophsical property of paraffin mixture (Chinese), Journal of Qingdao University of Science and Technology (Natural Science Edition), 35 (2014) 514-519.

[48] Z.Y. Liu, H.Y. Wu, Pore-scale study on flow and heat transfer in 3D reconstructed porous media using micro-tomography images, Applied Thermal Engineering, 100 (2016) 602-610.

[49] Y.T. Yang, F.H. Lai, Numerical study of flow and heat transfer characteristics of alumina-water nanofluids in a microchannel using the lattice Boltzmann method, International Communications in Heat and Mass Transfer, 38 (2011) 607-614. 